\title{
Extra Dimensions: 3D and time in PDF Documentation
}

\author{
Norman A. Graf \\ Stanford Linear Accelerator Center, Menlo Park, CA, 94025 \\ Norman.Graf@slac.stanford.edu
}

\begin{abstract}
High energy physics is replete with multi-dimensional information which is often poorly represented by the two dimensions of presentation slides and print media. Past efforts to disseminate such information to a wider audience have failed for a number of reasons, including a lack of standards which are easy to implement and have broad support. Adobe's Portable Document Format (PDF) has in recent years become the de facto standard for secure, dependable electronic information exchange. It has done so by creating an open format, providing support for multiple platforms and being reliable and extensible. By providing support for the ECMA standard Universal 3D (U3D) file format in its free Adobe Reader software, Adobe has made it easy to distribute and interact with 3D content. By providing support for scripting and animation, temporal data can also be easily distributed to a wide audience. In this talk, we present examples of HEP applications which take advantage of this functionality. We demonstrate how 3D detector elements can be documented, using either CAD drawings or other sources such as GEANT visualizations as input. Using this technique, higher dimensional data, such as LEGO plots or time-dependent information can be included in PDF files. In principle, a complete event display, with full interactivity, can be incorporated into a PDF file. This would allow the end user not only to customize the view and representation of the data, but to access the underlying data itself.
\end{abstract}

\section{Introduction}

We present a number of new features in the Adobe Portable Document Format (PDF) which could be used in HEP communications. In order to actually access the embedded three dimensional information, the user needs to use the latest version (at least version 8) of the Adobe PDF Reader[1]. Earlier versions, or third party PDF viewers, may not work correctly.

\section{3D and time in HEP}

As high energy physicists, we are used to using three spatial dimensions and time to describe the world around us. However, when documenting our results we are usually limited to the two dimensions of the printed word. The advent of electronic communication has made multimedia formats available, but the standard for documentation remains print, or print-like. This forces the author to make many compromises when presenting results. For instance, choosing the correct point of view for a reconstruction LEGO plot, or an event display, so that all the important information is visible, or showing multiple snapshots of a time sequence, can be very demanding. If the complete three-dimensional spatial information were accessible to the end user, many of these compromises could be avoided. 


\section{U3D: yet another "killer 3d app?"}

The Universal 3D (U3D) format is an ECMA standard[2] designed by the 3D Industry Forum. To quote from the Intel white paper[3], it aims to "simplify the transformation of complex 3D data into a format that can be streamed, compressed and viewed on affordable, non-proprietary software/hardware platforms while providing a high quality viewing experience.” This goal of providing three-dimensional, interactive content to a wider audience is not new. There have been many proposals in the past, VRML being perhaps the best known, which have aimed (and generally failed) to gain access to a broad audience. There are a number of proposals and projects under development, such as X3D. What sets this proposal apart is that it has been adopted by Adobe to serve as one of the 3D representations within the PDF file format, which is the de facto standard for document interchange today. Free readers are available on multiple platforms, and a number of programs are available which can convert files to this format. The Acrobat Reader, as of version 7, allows interaction with embedded 3D content. There already exists, therefore, a mechanism whereby data encoded in the U3D format can reach a wide audience.

\section{Interaction with 3D content}

One classic example of a three dimensional object which is difficult to easily render in two dimensions is the Möbius strip, shown in the following figure.

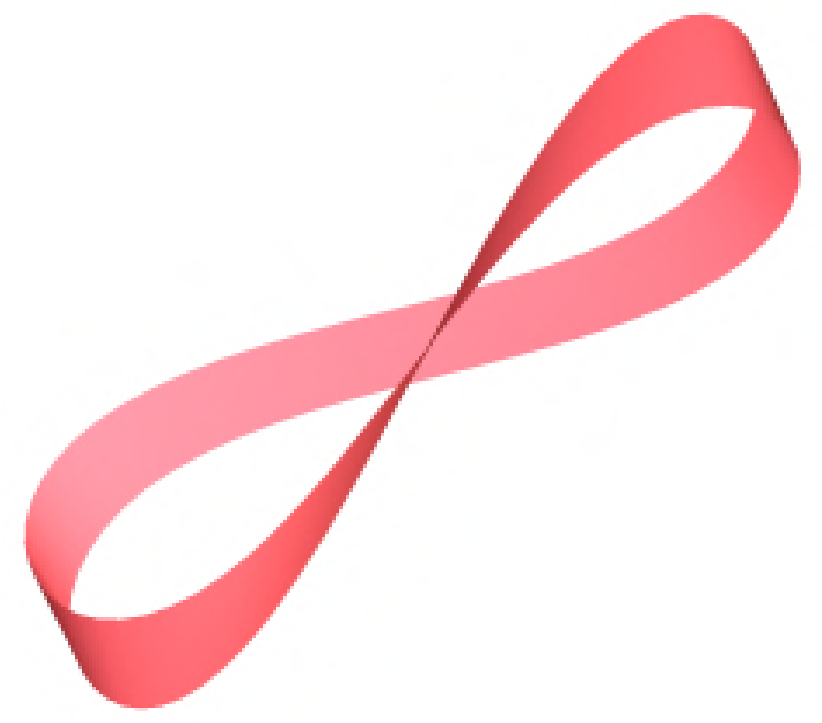

The Möbius Strip

By clicking on the above figure, you can activate the 3D interaction. This will bring up a toolbar similar to the one in the following (static) figure. You will then have the following options.

\& Rotate

- Spin

* Pan

Zoom

ล Default View 
Wears $\quad$ Select from predefined views (if any)

㫨 Toggle the model tree

Play Animation (if defined)

- Toggle between orthographic or perspective projections

Select from a number of model render modes (e.g. solid, transparent, illustration, outline, etc.)

9. Enable extra lighting

Set the background color

The Klein bottle is another higher-dimensional object where 3D interaction can enable the user to learn more and better understand the concept by interacting with it.

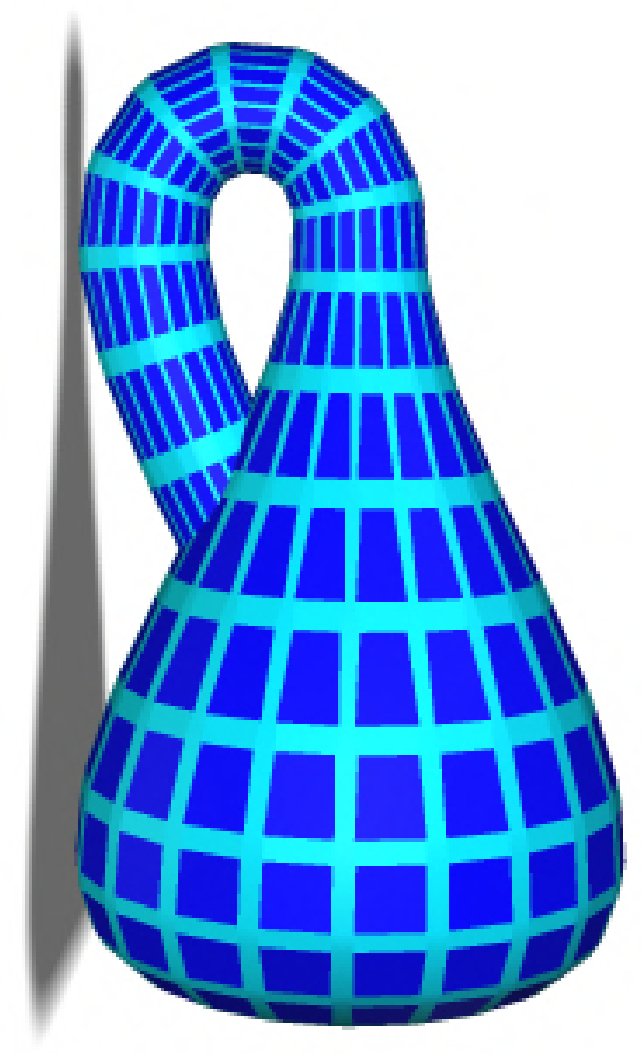

The Klein Bottle. 
Although these models demonstrate the utility of interaction with higher dimensional objects, they are not necessarily directly relevant to HEP. However, the same tools can be used to convey information about three dimensional fields, such as that from a magnetic dipole.

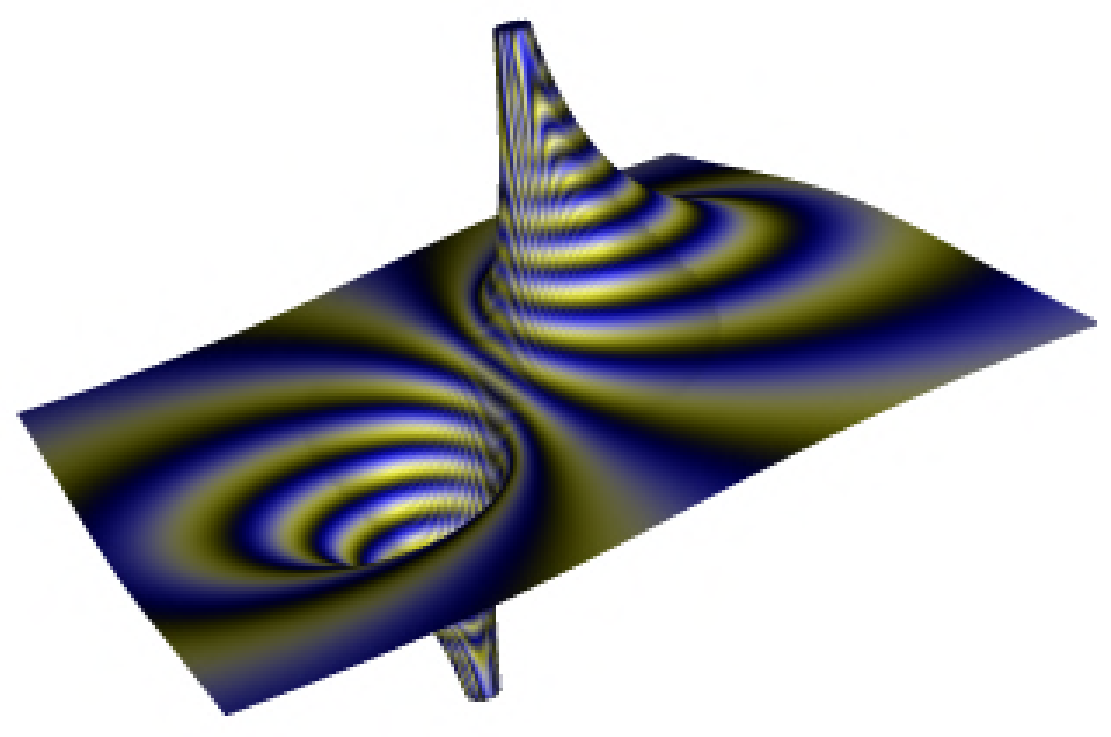

Magnetic Dipole Field.

This is a very high resolution map (roughly 80,000 faces), demonstrating the ability of the U3D file format to compress, and the Adobe Reader's ability to serve up, large arrays of information. 


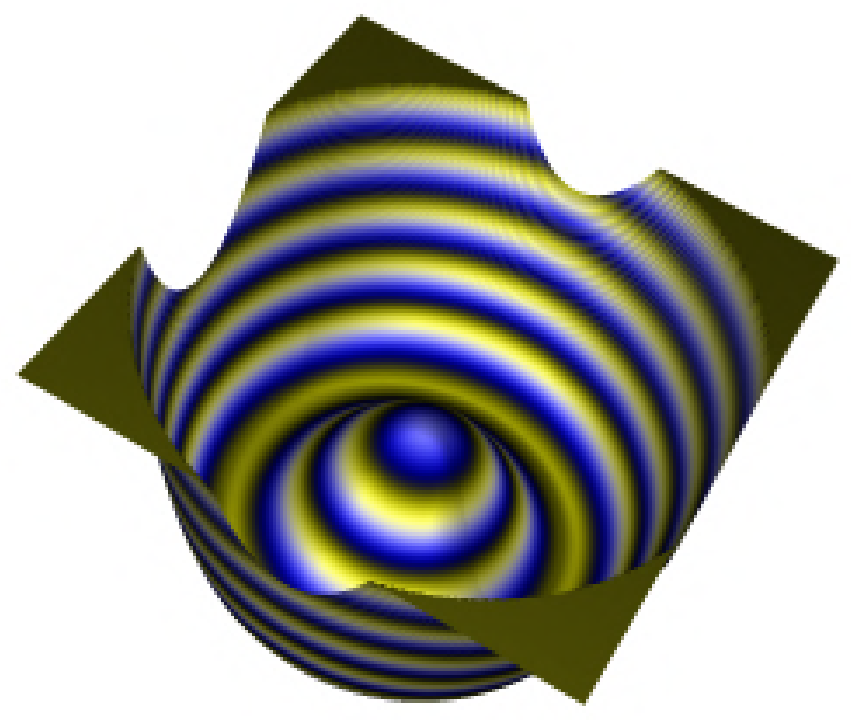

The Higgs Potential

Or, perhaps more relevant to HEP, the Higgs potential. In this image, there is a predefined cut view, showing the classic two-dimensional cross-section. However, this does not convey the sense of degeneracy nearly as well as being able to view the full, 3D potential map.

\subsection{Scripting}

PDF also provides support for JavaScript scripting. This allows hotspots in the text to be linked with views in an image. The following example, taken from the classic geometry exercise demonstrating conic sections, illustrates this. Clicking on the conic section names will bring up the corresponding views in the image.

\begin{tabular}{|l|l|l|l|l|l|}
\hline Parabola & Hyperbola & Circle & Ellipse \\
\hline
\end{tabular}

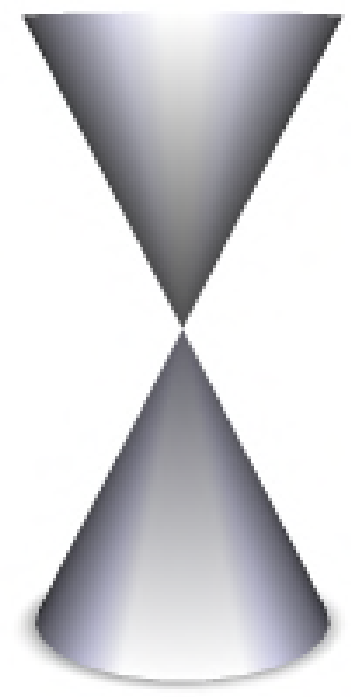




\section{Detector components}

Collaboration in the early stages of detector design is often hampered by either the inability to exchange CAD drawings between different proprietary software, or the inability of physicists to interact with or comment on the designs themselves. By embedding the 3D objects directly within a PDF file, all of the usual commenting and markup tools are available to the end user. As an example, we show a silicon microstrip module being designed for the International Linear Collider's Silicon Detector.

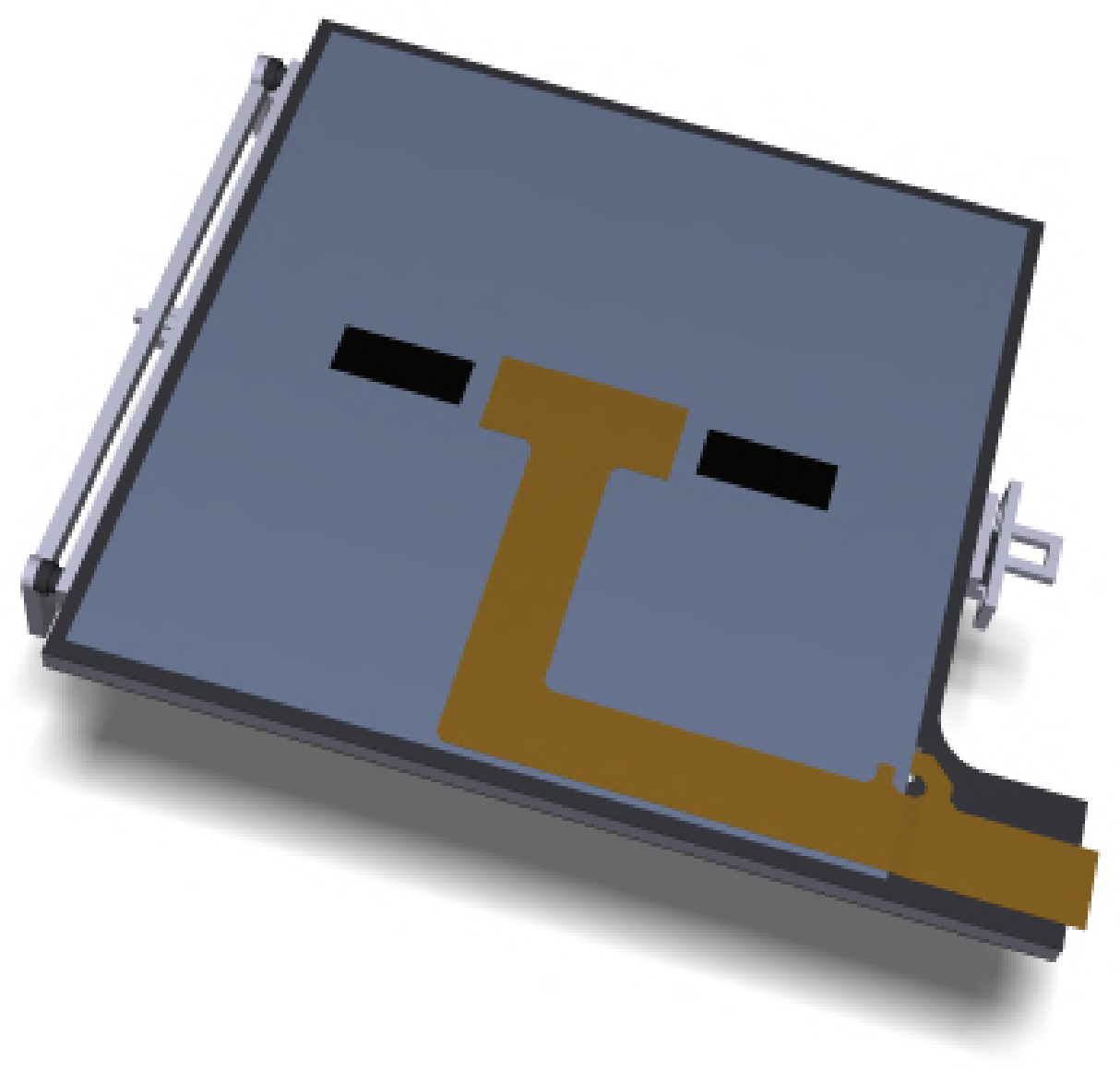

Conceptual design for silicon microstrip module.

A number of preset views are available for this object, as well as a detailed model tree and an animation timeline for the assembly process. 
The following images demonstrate some of the control which the viewer has over the display of this object simply by turning off various object elements, selecting different rendering modes, and changing lighting.
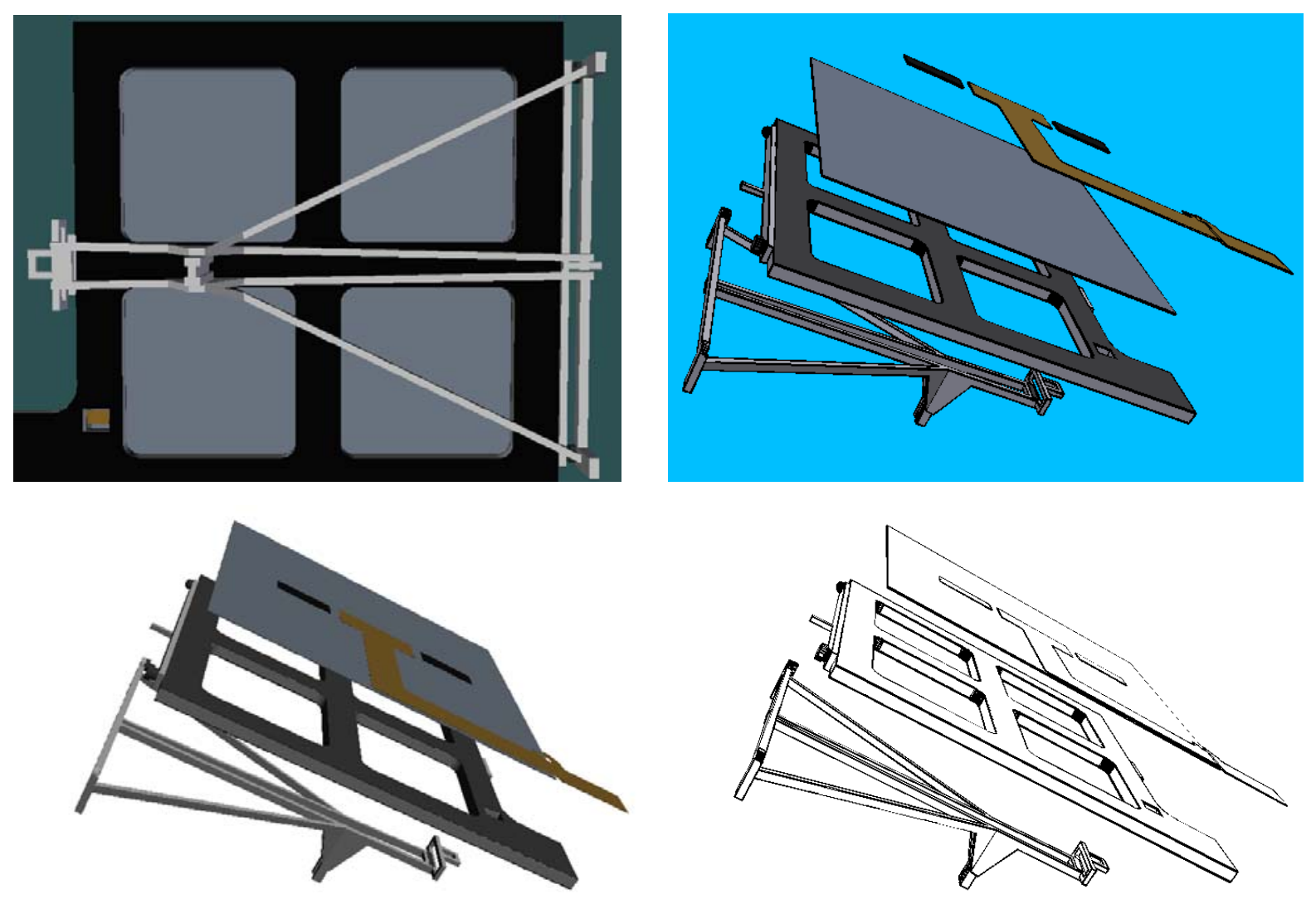


\section{Full detector description}

In the next figure, we include a full detector description, which the viewer is invited to explore using the tools and methods described above.

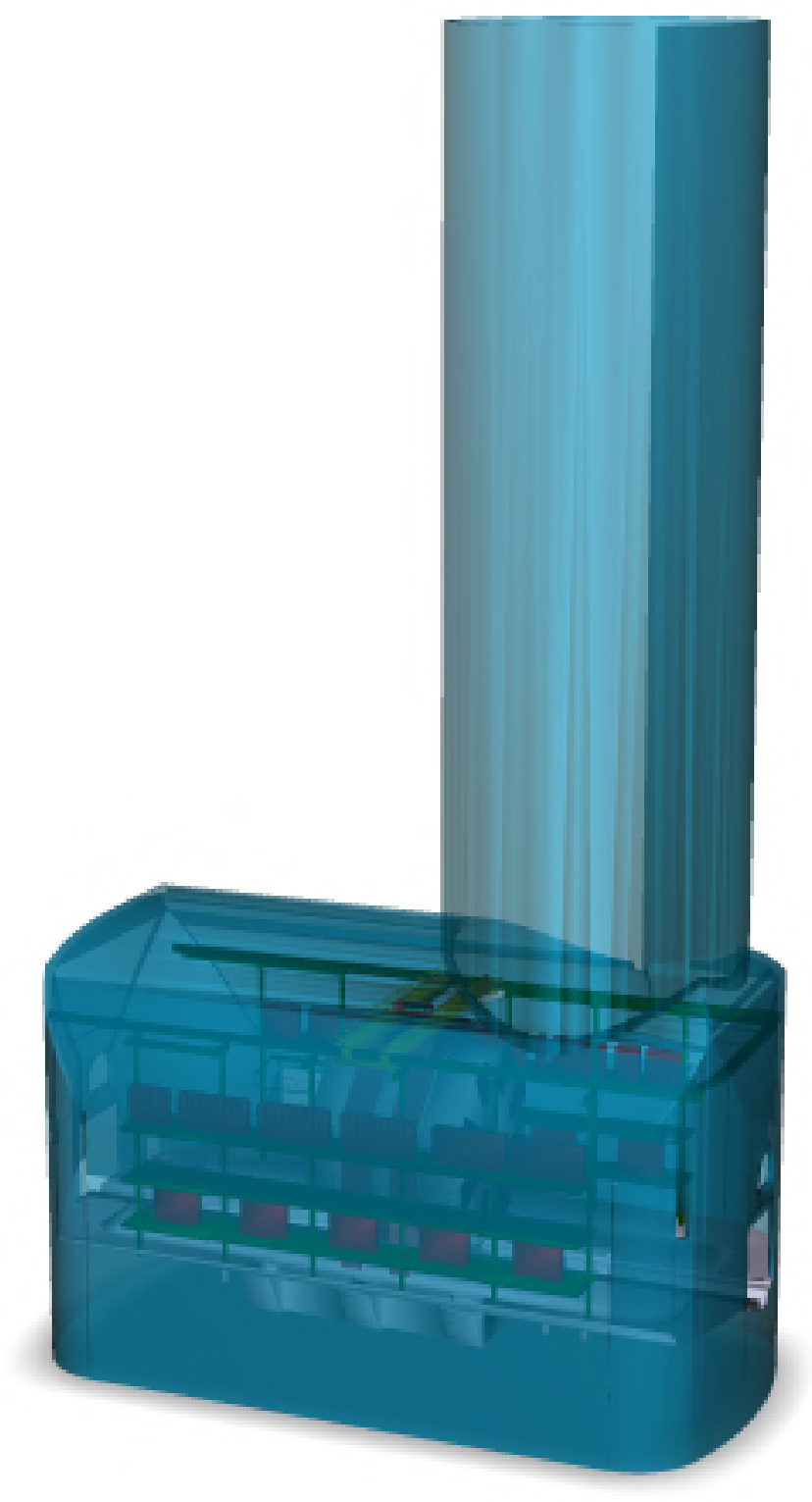

\section{Event display}

We are in the process of creating a binding to the U3D format which will allow us to embed full event displays from the data reconstruction. This will not, of course, replace any of the existing event displays which are accessible to developers and researchers within an experimental collaboration, but it will allow exceptional events to be fully documented and presented to a wider audience. The tools described above will allow such end users to customize the view of an event, turning on or off parts of the event or detector, customizing the representation of the view, and even gaining access to the fourvector information of the individual particles in the event. 


\section{Summary}

The ability to embed three dimensional objects and to enable time evolution of scenes or data in standard PDF documents opens up a whole new dimension (or two) of information transfer. End users, using freely available and widely supported readers on a number of platforms, have the ability to interact with the scenes, customizing the viewing experience, and can also exchange information via standard PDF markup tools. A number of HEP applications, ranging from documenting detector designs to exchanging reconstructed physics events, could benefit from this new functionality.

\section{Acknowledgements}

Work supported by Department of Energy contract DE-AC03-76SF00515.

\section{References}

[1] http://www.adobe.com/products/acrobat/readstep2.html

[2] http://www.ecma-international.org/publications/standards/Ecma-363.htm

[3] http://download.intel.com/technology/systems/U3D_WP.pdf 